\title{
PROGRAMA ÚNICO O DIFERENCIADO: ESPECIFICIDAD CURRICULAR DE LA ESCUELA RURAL URUGUAYA
}

\author{
Limber Elbio Santos \\ Universidad de la República, Uruguay.
}

\section{PROGRAMA ÚNICO OU DIFERENCIADO: ESPECIFICIDADE CURRICULAR DA ESCOLA RURAL URUGUAIA}

\begin{abstract}
Resumo
O artigo se refere ao fenômeno da especificidade curricular que as escolas rurais uruguaias tiveram durante mais de um século e à ruptura registrada em 2009 quando se começou a aplicar uma estrutura curricular comum a escolas urbanas e rurais. Analisam-se as circunstâncias históricas da pedagogia rural e, em particular, uma de suas materialidades simbolicamente mais potentes constituída pelo Programa para Escolas Rurais de 1949. Assim mesmo, refere-se ao peso que essa pedagogia continua tendo atualmente e da maneira como influi na gestão curricular do programa único. As especificidades social e didática da escola rural têm efeitos evidentes sobre o programa curricular no seu sentido mais amplo, com alcances muito mais extensos que os relativos ao currículo prescrito. No entanto, o desafio da escola rural atual, de abordar a nova estrutura curricular de caráter único, não é tão diferente do que se registrou historicamente: dialogar com o meio de igual para igual e maximizar o potencial de aprendizagem proporcionado pela instituição educacional, pela comunidade e estrutura multidisciplinar do grupo.

Palavras-chave: escola rural, currículo, programa, pedagogia rural.
\end{abstract}

\section{COMMON OR DIFFERENTIATED SCHOOL PROGRAMS: URUGUAYAN RURAL SCHOOL CURRICULUM SPECIFICITY}

\section{Abstract}

The paper refers to the phenomenon of the curriculum specificity to which the rural schools had for more than a century in Uruguay and to the rupture registered in 2009 when a common curriculum for rural and urban schools was introduced. The paper analyses the historic circumstances of the rural pedagogy and, in particular, one of its most potent symbolic materiality constituted of the 1949 program for rural education. The analysis show that the challenge to current rural education is not that different to that registered historically: to dialogue with the environment and take maximum advantage of the potential to learn which is provided by the education institutions, the community as well as the multi seried structure group.

Key-words: rural school, curriculum, program, rural pedagogy. 


\section{PROGRAMA ÚNICO O DIFERENCIADO: \\ ESPECIFICIDAD CURRICULAR DE LA ESCUELA RURAL URUGUAYA}

Resumen

El artículo refiere al fenómeno de la especificidad curricular que las escuelas rurales han tenido durante más de un siglo y al quiebre registrado en 2009 cuando se comenzó a aplicar una estructura curricular común a escuelas urbanas y rurales. Se analizan las circunstancias históricas de la pedagogía rural y en particular, una de sus materialidades simbólicamente más potentes, constituida por el Programa para Escuelas Rurales de 1949. Se hace referencia asimismo, al peso que esa pedagogía continúa teniendo en la actualidad y de qué manera influye en la gestión curricular del programa único. Las especificidades social y didáctica de la escuela rural tienen evidentes efectos sobre lo curricular en sentido amplio, con alcances mucho más extensos que los relativos al currículo prescripto. Por lo tanto, el desafío en la escuela rural actual de abordar la nueva estructura curricular de carácter único no es tan diferente del que se registró históricamente: dialogar con el medio de igual a igual y aprovechar al máximo el potencial para aprender que brinda la institución educativa, la comunidad y el grupo de estructura multigrado.

Palabras-clave: escuela rural, currículo, programa, pedagogía rural.

\section{PROGRAMME UNIQUE OU DIFFÉRENTIEL: SPÉCIFIQUE CURRICULAIRE DE L'ÉCOLE RURALE URUGUAYENNE}

Résumé

L'article fait référence au phénomène du programme de spécifique curriculaire que les écoles au milieu rurale ont eu depuis plus d'un siècle et la rupture enregistré en 2009 quand il a commencé à mettre en œuvre un programme commun aux écoles urbaines et rurales. Nous analysons les circonstances historiques de la pédagogie rural et en particulier, l'un des matérialités symboliquement plus puissants, constitué par le Programme pour les Écoles Rurales de 1949. Ainsi, il se réfère au poids que cette pédagogie poursuit actuellement et la manière à influencer dans la gestion curriculaire du programme unique. Les spécifiques sociale et didactique de l'école rurale a des effets évidents sur le programme curriculaire dans son sens le plus large, avec des gammes plus étendues que celles relatives au programme d'études prescrit. Cependant, le défi de l'école rurale actuelle coudre le nouveau structure curriculaire unique n'est pas si différent de celui enregistré historiquement: dialoguer avec le milieu comme un égal et maximiser le potentiel d'apprentissage offertes par l'établissement d'enseignement, la communauté et la structure multidisciplinaire du groupe.

Mots-clé: école rurale, curriculum, programmes d'étude, pédagogie rurale. 
- n Uruguay, durante todo el siglo 20 y hasta el año 2008 se aplicó un programa curricular diferenciado y específico para las escuelas rurales. Este proceso histórico implicó la aparición del emblemático Programa para Escuelas Rurales de 1949, producto del movimiento pedagógico rural desarrollado en el país durante esos años. Con variantes y paréntesis, ese programa estuvo formalmente vigente durante varias décadas, hasta que en 2009 comenzó a aplicarse, por primera vez en más de 100 años, una estructura curricular común parta escuelas urbanas y rurales.

Hagamos un recorrido histórico por la construcción de la pedagogía rural uruguaya en torno al programa de 1949 como una de sus materialidades esenciales, para después considerar las circunstancias actuales de la escuela rural frente al programa único. Veamos, en este sentido, el significado pedagógico que continúa teniendo aquel programa y la manera en que la pedagogía rural puede generar en la actualidad, nuevos criterios para la gestión curricular sobe un programa sin diferenciaciones.

\section{La pedagogía rural uruguaya ${ }^{1}$}

Cuando analizamos el rol de la escuela rural uruguaya en la sociedad actual hacemos referencia inevitablemente a los elementos que conformaron una escena fundante de construcción pedagógica. En Uruguay, el medio rural de la década de 1930 dio lugar a un pensamiento, a una generación y a una producción intelectual de maestros que en ese momento estaban trabajando en el medio y que, como producto de ese proceso, construyeron una pedagogía rural. Una escena fundante en la que la visualización del rol de la escuela rural estuvo, en principio, muy de la mano de lo que constituía el estrecho vínculo que la escuela rural tenía con las comunidades, con las familias, con el entorno y con la sociedad rural en la cual estaba inserta. Sin duda que aquella sociedad rural generó el pensamiento de los maestros de la década de 1940 y que luego claramente se visualiza en algunos productos de aquel proceso como son los fundamentos, conceptos y fines que estuvieron presentes en la estructura curricular que se construyó y que estuvo vigente en la educación primaria rural en términos formales hasta 2008. Allí hay conceptos que han sobrevivido hasta la actualidad porque son bastante más profundos que el marco curricular en el cual se incluyeron.

En esa escena fundante la escuela rural estaba ubicada en un contexto caracterizado, sobre todo, por la presencia de los rancheríos rurales, de los cuales daba cuenta Julio Castro. Allí la escuela era la única institución pública, la única presencia del Estado, ubicada en el marco de esa conformación social con tantos problemas e identificada en ese momento con tantas realidades a lo largo y ancho del país. Lo que se comienza a estructurar como pensamiento en torno al rol de la escuela rural es un discurso que a partir de la década de 1930 se comienza a desarrollar en forma de denuncia. Denuncia de parte de los maestros acerca de las condiciones de las escuelas rurales ubicadas en ese contexto. De ahí aquella expresión del maestro Pedro Ferrari en 1933, que se refería a la casucha destartalada que hacía las veces de escuela y que casi no se diferenciaba del medio que la rodeaba. Era la denuncia de las condiciones reales y concretas de vida que los uruguayos sufrían dentro de los rancheríos rurales.

\footnotetext{
${ }^{1}$ Este y los próximos apartados vinculados a la pedagogía rural uruguaya, sus circunstancias históricas y el Programa para Escuelas Rurales de 1949 como materialidad pedagógica, están basados en Santos (2011). 
De algún modo, el magisterio rural tuvo un papel muy importante, en tanto puso sobre la mesa la discusión acerca de ese Uruguay que desde Montevideo - sobre todo en el Uruguay de la década de 1940, de la posguerra y aletargado por la bonanza económica de ese período - no se veía o no se quería ver. Julio Castro fue claro al regresar de la misión sociopedagógica de 1945 a Caraguatá al denunciar con crudeza lo que era un rancherío rural desde adentro y cómo era ese Uruguay donde no solo vivían los habitantes de Caraguatá, sino 130.000 uruguayos más en varios departamentos del interior. La denuncia de los maestros y de los estudiantes magisteriales en 1945 en las contratapas del semanario Marcha entre julio y agosto de ese año, durante la misión y a su regreso de Caraguatá, tuvo un fuerte impacto. Vino a acelerar un proceso que ya se había iniciado por parte del magisterio en 1933, poniendo sobre la mesa el tema de lo rural y el papel que debía cumplir la escuela en ese entorno. Eso supuso abrir una discusión que dentro del magisterio ya se venía dando y tenía un proceso de casi 15 años de trabajo, de procesamiento, de instancias de discusión y de producción intelectual que circulaba entre los maestros a través de los órganos de difusión, gran parte de ellos oficiales.

El hecho de ubicar el tema en la prensa, ubicarlo en el debate público y en la opinión pública, presionó sobre los poderes políticos para que se tomaran algunas decisiones acerca de ese Uruguay. La generación del producto pedagógico de ese proceso fueron los fundamentos, concepto y fines de la escuela rural en el Programa para Escuelas Rurales (1949). Una estructura curricular pensada específicamente para las escuelas rurales que en 1949 viene a contemplar la especificidad para tomar lo mejor de sí y aprovechar el potencial existente en la escuela y en el medio rural, para que eso jugara a favor y no se considerara como un mal necesario o como una situación carente para la cual se ofrecía una educación de menor calidad. A partir de 1949 se llega a un consenso con respecto al lugar que debía tener la escuela en relación a las comunidades rurales, con efectos concretos entre los propios maestros organizados y las autoridades de la educación del momento. Esto no fue casual, fue producto de esa presión que los propios maestros ejercieron sobre el poder político para que se tomaran decisiones acerca de la situación del medio rural y la situación de la escuela en ese medio.

Todo lo que se genera después, en la década de 1950, se corresponde con ese pensamiento. Hubo una etapa, corta en el largo período de la historia de la educación pública uruguaya, pero muy potente, simbólicamente muy importante, cuyos efectos se sienten todavía hoy como herencia de esas acciones. Fueron acciones que se correspondieron con el pensamiento de la impronta social muy fuerte de la escuela rural, el pensamiento de construir una pedagogía que tuviera elementos de lo educativo pero también de lo social, al mismo nivel. Pensamiento que derivó en el concepto de escuela productiva como la escuela que generara productos educativa y socialmente útiles, que para el magisterio rural está muy naturalizado pero que, sin embargo, era un concepto muy potente a fines de la década de 1940, cuando se equiparaba la función educativa y la función social en torno a los productos que la escuela rural pudiese generar. La cuestión del rol en relación a lo social de la escuela rural era un componente muy fuerte de esa pedagogía y, de algún modo, constituía la identidad propia y característica de ese proceso de discusión. 
Allí había una tensión. La atribución del rol vinculado con lo social a la escuela rural, basado en su especificidad social, en tanto única institución físicamente presente en la mayor parte de las comunidades rurales, determinaba un rol que estaba en ese Programa de 1949 bajo la forma de fines sociales. Fines sociales que eran revolucionarios: atribuir a la escuela el fin social de ser el centro de la comunidad, ser la "casa del pueblo porque es al casa de los hijos del pueblo", en palabras de Julio Castro; constituirse en el lugar de confluencia de las acciones para el mejoramiento de la sociedad rural. Un objetivo extremadamente ambicioso que, sin embargo, luego se llevó a la práctica en experiencias como las del maestro Soler en La Mina, donde precisamente el gran objetivo era "ayudar a vivir mejor" (Soler, 2005, p. 232).

Ubicar ese objetivo como parte de lo que debía hacer la escuela era algo ambicioso. Y allí estaba instalada una tensión que no era nueva, que ya se había iniciado en 1933 en el pensamiento de los maestros y que tenía que ver con el rol que debía ocupar la escuela en la sociedad rural. La tensión estaba ubicada en cómo la escuela como institución educativa podía contribuir al cumplimiento de fines tan ambiciosos como ayudar a vivir mejor y ayudar a transformar la sociedad rural. Los maestros rurales fueron muy claros en ese momento y lo han sido a lo largo de toda la historia. No así las políticas educativas que han tenido distintas etapas: a aquella etapa de coincidencia en 1949 luego le sobrevinieron otras etapas de discrepancia y hasta de contraposición con el pensamiento del magisterio respecto a este punto.

Vincular tan fuertemente a la escuela con el cumplimiento de fines sociales estaba en 1933 ya ubicado en el justo lugar por parte de los maestros cuando rechazaban la idea de que la escuela por sí sola podía transformar la sociedad rural. Ese año los maestros fueron convocados para discutir una problemática que ya preocupaba: la emigración del campo a la ciudad. Se conformó una comisión de trabajo para discutir qué puede hacer la escuela para evitar la despoblación del campo. La respuesta de los maestros pasó por la posibilidad de contribuir a los procesos de cambio, pero sin dejar a la escuela en solitario para que resolviera un problema de índole estructural y multicausal en el que deben intervenir otras esferas del Estado y de lo público para que, en conjunto con la escuela, puedan actuar en esa problemática. Y el emblema que simbólicamente surge de 1933 es la escuela sola no puede. Esa misma idea es la que está presente en el final de ese proceso en 1949 y que da lugar a una nueva etapa en la década de 1950. En el Programa de 1949 ese rol atribuido a la escuela desde lo social va de la mano de la explicitación de los límites de la educación.

Eso es lo que se plasma en el Núcleo Escolar Experimental de La Mina, que es el ejemplo práctico que más se identifica con el planteamiento teórico de 1949. "Ayudar a vivir mejor" es el gran objetivo. Sin embargo, ese objetivo no se puede lograr si la escuela no actúa en conjunto con otras instituciones y es claro que la experiencia implicaba la intervención de distintas instituciones, actuando en lo que a cada una le correspondía, teniendo a la escuela rural como punto de confluencia. Allí estaba la resolución de esa tensión, que no desaparece, pero que se resolvía negando la idea de que la escuela se debía encargar de todo y que el maestro debía hacerlo todo. La escuela es una institución educativa, y en ella y en la figura del maestro confluye un esfuerzo interinstitucional.

Sin duda que aquel marco en el que se ubica ese rol particular de la escuela rural con respecto a lo social tenía dos conceptos fuertes en esa pedagogía. Uno de ellos era 
el concepto de medio. Un concepto simple pero muy potente. Está presente en esa pedagogía como el medio enseñante, el medio con el cual la institución educativa interactúa de dos maneras: extrayendo de ese medio lo que éste tiene para incorporarlo a la propuesta de la escuela, pero en una tensión que implica también que la escuela cumpla un papel específico para compensar los déficits que ese medio posee. De algún modo, tener en cuenta los elementos del medio como punto de partida implicaba también trascenderlos y ofrecer aquello que el medio no podía ofrecer, en una relación de diálogo de igual a igual. Ese concepto de medio, muy diluido en las últimas décadas, ha sido asimilado a otros conceptos como el de contexto, de connotaciones absolutamente distintas, siempre vinculado a un contexto crítico no enseñante y del cual hay que defenderse, distanciándose bastante de aquella vieja concepción.

El segundo concepto, el de escuela productiva ${ }^{2}$, implicaba que los dos grandes requisitos para los productos de la escuela, ubicados de igual a igual, el estrictamente educativo y el social, estuviesen presentes como signo de la labor cotidiana de la escuela.

El maestro Miguel Soler, en el Primer Coloquio de Educación Rural en Montevideo $(2009)^{3}$, observaba cómo la diversidad enorme de experiencias actuales que se expusieron por parte del equipo del Departamento de Educación para el Medio Rural, representativas de todos los departamentos del país - sencillas pero muy potentes, emanadas de la vida cotidiana de las escuelas rurales actuales -, refieren a acciones que ya se hicieron antes. Acciones que, con la excepción del Plan Ceibal, en algún momento en el país tuvieron lugar, se dejaron de hacer y él veía con beneplácito cómo ahora se estaba retomando ese camino. Y había varios ejemplos de eso en las experiencias que se compartieron en el coloquio.

El magisterio rural nunca abandonó la matriz pedagógica que generó aquel movimiento. Se han registrado etapas muy duras, en dictadura pero también en democracia, durante las cuales los maestros rurales han resistido por distintos mecanismos. En la década de 1960, con la creación del Instituto Cooperativo de Educación Rural (Icer), para seguir reivindicando por parte de los propios protagonistas de aquel movimiento de la década de 1950 lo que constituía aquella pedagogía. En tiempos más recientes, en la década de 1990, creando instancias propias de definición de la posición política, técnica y pedagógica del magisterio rural, explicitada a través del Congreso de Maestros Rurales de 2005 y los Encuentros de Maestros Rurales de las Asambleas Técnico Docentes de 2003 y 2005. En esas expresiones nunca se abandonó la matriz pedagógica de aquellos años, actualizándola a la luz de la realidad actual.

\section{El currículo diferenciado para escuelas rurales ${ }^{4}$}

\footnotetext{
${ }^{2}$ En el texto del Programa para Escuelas Rurales que los maestros rurales elaboraron en 1949 se planteaba el concepto de Escuela Productiva en estos términos: "La Escuela no será productiva si la producción se entiende como exclusiva creación de bienes económicos. Será productiva, en cambio, si la producción se entiende como trabajo educativa y socialmente útil, que pueda crear beneficios materiales para los alumnos".

${ }^{3}$ En diciembre de 2009 se realizó en Montevideo el Primer Coloquio de Educación Rural, organizado por el Departamento de Educación para el Medio Rural del Consejo de Educación Inicial y Primaria, que luego tuviera otras tres ediciones en Cruz de los Caminos, en 2010, 2011 y 2012. Se trató de instancias de difusión de experiencias educativas en escuelas rurales de todo el país, en lo técnico docente, lo socio comunitario y la coordinación interinstitucional.

${ }^{4}$ Este y los próximos apartados sobre el currículo diferenciado de 1949 y el currículo común de 2008 están basados en Santos (2009). 
El producto pedagógico de mayor peso simbólico en la historia de la educación uruguaya es el Programa para escuelas rurales de 1949. Derivado de un esfuerzo conjunto de los maestros rurales y producto de un largo proceso de reivindicación política y técnica, el documento emanado del Congreso de Piriápolis del mes de enero y aprobado en octubre de aquel año, representa como estructura curricular, un conjunto de concepciones sobre la educación en general y sobre la escuela rural en particular. Si bien de aquel texto lo único que se ha mantenido inalterable es el exordio conocido como Fundamentos, concepto y fines de la escuela rural, todos los maestros aún hoy identificamos el Programa rural con aquel viejo Programa del 49. Pero, como ha dicho el Maestro Miguel Soler - único sobreviviente de los integrantes de la comisión redactora en aquel processo -, las modificaciones estructurales introducidas en la revisión de 1987, modificaron de tal manera la propuesta original, que ya nada justifica esa identificación.

Sin embargo, los tiempos actuales marcan ahora sí, un quiebre en la continuidad histórica, en lo que se refiere a las propuestas curriculares para la escuela rural. Desaparece del escenario educativo, la diferenciación curricular entre escuelas urbanas y rurales, tras muchas décadas de construcción pedagógica y realización de prácticas educativas, a la luz de esa diferenciación. Hecho nada menor, si tenemos en cuenta además, que dicha diferenciación no fue producto del ya conocido proceso que culminó en 1949, sino que ya se registraba desde 1917 y aún antes, desde 1897; por lo que ha sido un elemento que ha caracterizado nuestra escuela pública desde hace más de 100 años. Aunque no resulte baladí, esta transición no supone tampoco, una catástrofe imposible de superar. Por el contrario, con juicio crítico pero a la vez con espíritu prospectivo, los maestros rurales debemos poner en juego lo mejor de nuestra realidad, para adecuar la nueva propuesta curricular a sus características y especificidades que siempre hemos defendido. Sea como fuere, el currículum prescripto siempre cede ante la presencia de la realidad, en términos de priorización y adaptación, y necesitarán por parte del magisterio rural, una serie de acciones y producciones ad hoc, que hagan posible esos mecanismos.

\section{El sentido de la especificidad}

Un programa específico para escuelas rurales implicaba aprovechar lo mejor del medio donde la enseñanza y el aprendizaje tenían lugar, con el propósito de formar plenamente un niño que luego hombre pueda desarrollarse adecuadamente en ese medio que es el que le toca vivir, o en cualquier otro donde la vida le lleve. A esa altura se manejaba la convicción de que la circunstancia de si el niño se quedaba en el medio rural o emigraba a otros lugares en el futuro, no dependía sólo de la escuela. Ya había corrido mucha agua debajo de los puentes y la fermental discusión de los maestros rurales de los años 30 y 40 había alejado de la consideración pública el hecho de que la escuela pudiese resolver sola todos los problemas de la sociedad. La escuela sola no puede había sido la consigna resultante de la discusión de los maestros en el Congreso de 1933, reclamándose de otras esferas públicas la contribución a intervenir en la solución a los problemas sociales. De ahí que en los referidos fundamentos del Programa de 1949 se hagan referencias a los condicionamientos políticos y económicos de la educación, sosteniendo que el supuesto de que las reformas educativas pueden cambiar la sociedad es "antinatural y antihistórico" (CPEPyN, 1950, p. 11). Esa prudencia conceptual llevó a 
adoptar una postura cauta con respecto a la importancia del Programa escolar: imprescindible, pero mínima si no está acompañada de una serie de acciones que, en el caso del medio rural, posibiliten adecuar la formación de los docentes para un máximo aprovechamiento de los recursos diferenciales disponibles.

El resultado de esa posición fue la creación de un Programa cuya diferencia central con respecto al urbano, nunca estuvo centrada en una diferencia de contenidos a enseñar, como podría suponerse y en lo cual se basaban las críticas iniciales. Si bien había diferencias en cuanto a la enumeración de contenidos, la diferencia fundamental estaba vinculada con la consideración de los puntos de partida: los recursos disponibles y la manera de aprovecharlos. Se trataba de materializar los reclamos formulados por Agustín Ferreiro desde hacía más de una década, en términos de adecuación a la realidad; esto es, atender lo que el niño y su familia ya poseen y conocen, aprovechar lo que lo que se presenta alrededor del acto educativo en la escuela rural y que por cierto, es bien distinto de lo que se presenta en un ámbito urbano. A esa primigenia reivindicación de Ferreiro se le suma en los años 40 y tras los oscuros diagnósticos del estado de la escuela rural de entonces, la reivindicación de pretender para los niños rurales una educación de igual o superior calidad que la de los niños urbanos. De la síntesis de ambas convicciones, surgió un Programa que mantenía la diferenciación curricular como desde 1917, pero a partir de una propuesta de avanzada, con fuertes contenidos universales sustentados en una estructuración, una lógica y unos puntos de partida, específicamente concebidos desde y para el medio rural.

\section{La tensión entre lo general y lo particular}

El conjunto de conocimientos que es designado para ser enseñado, en tanto recorte del universo de conocimientos disponible, supone una serie de decisiones políticas y técnicas, cuyo producto es el currículum. Si bien el currículum prescripto manifestado en el Programa, es una parte importante de esas decisiones, no constituye lo único. El conjunto de prácticas, tradiciones y usos habituales presentes en los acontecimientos de enseñanza, constituye también la estructura curricular, gran parte de la cual permanece invisible, bajo la forma de currículum oculto. En ambas esferas, la que enumera contenidos en el programa y la que determina su enseñanza a través de aquello que no necesariamente se explicita; se manifiesta una tensión entre lo general y lo particular. Se trata de un debate que formó parte de la discusión de los años 40 en nuestro país, a propósito de la conformación de una estructura de educación rural en el sistema de educación público, incluyendo políticas educativas diferenciales y un programa específico. Se trata del debate acerca de cómo debe estar configurado el currículum, contemplando un corpus general de conocimiento - universal, nacional - y un conjunto de saberes locales, vinculado con la región, la comunidad y hasta el propio centro educativo.

Las características de nuestro país no ha determinado decisiones de política educativa que habiliten incorporaciones locales o regionales al currículum prescripto. Sí ha ocurrido que los ámbitos rural y urbano han sido objeto de diferenciación que no es territorial estrictamente, sino que responde al entendimiento de que entre ellos hay una diferencia sustancial de idiosincracias, modos de vida y necesidades educativas. Esto quedó así establecido bajo fundamentos sólidamente explicitados en toda la producción pedagógica previa a 1949, a partir de los aportes iniciales de Ferreiro y los diagnósticos y 
propuestas de Julio Castro más tarde. Esto a pesar de las muy pertinentes y necesarias apreciaciones del propio Castro formuladas en 1940 acerca de la inexistencia de un medio rural, explicitando las diferencias entre los medios rurales ganaderos del norte y los medios agrícolas del sur.

El programa de 1949 tuvo el mérito de equilibrar una propuesta que contemplara una serie de conocimientos generales que se entiende la escuela pública debe enseñar y una serie de manifestaciones -mínimamente explicitados bajo la forma de contenidos y máximamente bajo la forma de sugerencias, objetivos y fundamentos- que buscan su adecuación al medio rural. Visto en su conjunto, el texto curricular persigue una formación integral del individuo en relación a otros y a la comunidad a que pertenece. La tensión entre lo general y lo particular es resuelta a través de la consideración de los insumos del medio - un medio enseñante- como punto de partida; a la vez de ofrecer lo nuevo, lo diferente, lo universal para compensar los déficits de ese medio. Es un texto que cumple con la contextualización de los conocimientos a ser enseñados, en tanto operación de vinculación con lo que ya está existe y es vivido; pero a la vez cumple con el necesario extrañamiento y ajenidad que todo conocimiento a ser enseñado debe tener.

Todo había comenzado con aquella imagen de Ferreiro de 1936; la que expresaba que enseñar en la escuela a plantar papas de igual o inferior calidad que las que ya se plantan por parte de los vecinos de la zona, no tiene sentido. El sentido está cuando la escuela se convierte en un humilde campo de experimentación proponiendo una técnica de producción nueva o una forma diferente de trabajo. Ese necesario extrañamiento de los conocimientos a enseñar se mantendría en el propio Programa, aún cuando a la vez, en sus fundamentos se propendería a evitar que la escuela rural fuese considerada un lugar de excepción. Allí, el rol social de la escuela como referente de la comunidad y parte indisoluble de la misma, se equilibraría con la naturaleza de los conocimientos a ser enseñados.

\section{La transición hacia el programa único}

La forma cómo se resolvió la tensión entre lo general y lo particular en la estructura curricular de la escuela rural, facilita las cosas. El carácter abierto del Programa para hacer que la escuela no sea un lugar de excepción, pero a la vez logre trascender el medio que le rodea; habilita a que hoy en día, se puedan buscar los mismos objetivos, desde un programa único. Esto no supone renunciar a la especificidad pedagógica de la escuela rural ni a su singularidad social y didáctica. Supone encontrar los mejores caminos para seguir defendiendo esos elementos, ante el nuevo escenario. Con programa único o sin él, la realidad didáctica alimentada por la estructura curricular, seguirá teniendo las complejas y ricas características de los grupos multigrado y la particular realidad social de la campaña. Aunque la transición pueda estar marcada por la frustración ante la evidencia de las ausencias, de aquellos contenidos que los maestros rurales entienden debieran estar en el programa y no lo están; la tarea didáctica y los fundamentos pedagógicos deben seguir la misma línea. El nuevo programa no lo impedirá. El desafío de la transición es aprovechar lo mejor de él y poner en juego el peso de la tradición pedagógica rural, para compensar sus eventuales déficits y lograr traducciones prácticas verdaderamente significativas y originales. 


\section{Viejas y nuevas características}

El movimiento pendular de las tendencias en educación ha provocado una vuelta a la enumeración de contenidos disciplinares, en reacción a enfoques integradores de contenidos que suponían, entre otras modalidades, su agrupación en torno a temas y problemas de la realidad. Pero esa agrupación es más propia de los dispositivos didácticos que de las estructuras curriculares prescriptas. En estas últimas, esa intención integradora no es reciente sin embargo. Los propios programas para escuelas urbanas de 1957 y para escuelas rurales de 1949 agrupan contenidos disciplinares en el área de las ciencias naturales. Una forma de estructurar el currículum en este sector del conocimiento que en su traducción en prácticas de enseñanza puede ser tan compartimentada como antes, pero que al menos, pretende prescribir lo contrario desde el currículum. Una manifestación particular de este fenómeno integrador de contenidos lo constituye la existencia en el Programa para escuelas rurales del área de "seres y fenómenos de la naturaleza", ya que no se corresponde en absoluto a denominación epistémica alguna, apelando a la experiencia y vivencias del medio donde esos contenidos vinculados con la biología y en menor medida con la química y la física, van a ser enseñados. Es interesante porque supone explicitar en la estructura curricular, un adelanto de cómo se manifiestan los contenidos educativos en los procesos de enseñanza.

El enfoque del nuevo programa resalta la importancia de los contenidos en relación a las estructuras disciplinares de las que surgen en el ámbito de la ciencia; configurando una reacción contra no sólo las tendencias integradoras en torno a grandes áreas (ciencias de la naturaleza, ciencias sociales) propias de los procesos de reforma de la educación secundaria de hace algunos años, sino que también alcanza las manifestaciones que en ese sentido tienen los viejos programas escolares.

Allí están los contenidos de seres y fenómenos de la naturaleza -los de siempre y muchos nuevos - pero expuestos conforme a una clasificación por disciplinas. Hay además, un grado de exhaustividad conceptual mucho mayor, como consecuencia del énfasis puesto en la lógica disciplinar. Pero esas características, los problemas visualizados a partir del volumen de contenidos expuestos y el alejamiento de los éstos respecto a las características del medio rural, debieran ser abordados a partir del cruce de la estructura curricular y la dimensión pedagógica primero y didáctica después, propias de la escuela rural.

\section{Miradas desde lo pedagógico}

Los contenidos del nuevo programa, aplicados en el medio rural, estarán atravesados por los principios pedagógicos históricamente construidos en relación a la ubicación de la institución escolar en ese medio. Estos principios no desaparecen con el nuevo programa, ya que trascienden lo curricular. Se trata de principios pedagógicos que, si bien requieren nuevas lecturas a la luz de la ruralidad actual, en esencia se mantienen vigentes, en tanto expresan las dos especificidades de la escuela rural. La primera de ellas refiere a la especificidad social, la que se deriva de la ubicación de la escuela en el medio como única institución pública y como única presencia del Estado. Elementos nada menores y que tienen efectos sobre el papel que la institución debe asumir, necesariamente. 
La segunda se refiere a la especificidad didáctica, la que se deriva de la existencia del grupo multigrado, con todas las consecuencias que ello tiene sobre los procesos de enseñanza y aprendizaje. Sin dudas que ambas especificidades, - la primera de ellas fuertemente defendidas en el proceso de creación del Programa de 1949 -, siguen teniendo hoy aún más razón de ser que antes. Esto puede evidenciarse en el proceso de despoblación del campo que se ha producido en estas décadas, lo cual ha resignificado la relación de la escuela con ese entorno por un lado y a la vez, ha provocado un aumento de los grupos multigrado en escuelas que progresivamente han visto reducir su matrícula.

Seguir teniendo en cuenta estas especificidades supone efectuar este cruce del texto del nuevo Programa con esa pedagogía de la educación rural. Más allá de las omisiones, leer el Programa desde esta clave implica encontrar los puntos de partida para su uso en el medio rural. Para ello será válido una vez más, el estar atento a los recursos que brinda el medio así como a las necesidades que se imponen allí, de lo cual otra vez, los contenidos referidos a las ciencias de la naturaleza, podrán ubicarse en el lugar central o al menos, estructurador de la traducción del currículum en prácticas de enseñanza.

La lectura del programa desde la escuela rural no implica su contextualización. Implica, como ya hemos dicho, encontrar vínculos entre los contenidos que están para ser enseñado y la realidad vivida y percibida, como un punto de partida que debe ser trascendido con los acontecimientos de enseñanza y aprendizaje. Esto supone el doble juego de valorar el medio rural - su cultura, sus prácticas sociales, sus formas de producción, sus dinâmicas - y al mismo tiempo cumplir con la función de enseñanza, en tanto acercamiento a lo lejano, extraño y ajeno. La relación con los saberes que se enseñan e intentan aprenderse, en parte debe ser una relación de extrañamiento, un extrañamiento ubicado en las coordenadas donde esos procesos tienen lugar. Se podría decir en este sentido, que la clave está en la consideración del currículum situado en los parámetros ambientales y didácticos donde tendrá su traducción en prácticas de enseñanza. El texto y su presentación no tendrán variaciones y tendrán un carácter universal, la lectura que hagamos de él sí deberá variar y tendrá una naturaleza singular.

\section{Miradas desde lo didáctico}

En la pedagogía rural, el puente entre la especificidad social y la especificidad didáctica, ha estado pautado por el uso de los recursos humanos y materiales del medio. La transformación de esos recursos en recursos didácticos por la decisión de los docentes, traza uno de los caminos que conduce a la didáctica en escuelas rurales. Las características particulares de los grupos multigrado es uno de los caminos que conduce a la construcción de una didáctica multigrado. En esta construcción, hemos considerado que el factor clave es la organización de los contenidos, basándonos en una didáctica centrada en los saberes. Si esto era así hasta ahora, mucho más lo será a la luz del nuevo programa con su fuerte énfasis conceptual. Por lo tanto en la organización de los contenidos se centrará el cruce del Programa con lo didáctico en la escuela rural, a partir de los temas estructuradores que el propio Programa explicite o los que emerjan en el aula y su entorno.

El grupo multigrado habilita la circulación de los saberes en términos didácticos, a partir de una cierta organización de los saberes en términos epistemológicos. Esa 
organización es igualmente didáctica, si adoptamos una didáctica epistemologizada, esto es, que tenga como centralidad a los saberes, su naturaleza, sus transformaciones y su circulación.

Para centrarse en los saberes y sus posibilidades de organización, es necesario contar con una sólida formación disciplinar. Eso permitirá conocer las lógicas que indican la mejor manera de integrar contenidos en torno a ejes estructuradores comunes, a partir de criterios de jerarquización y complementariedad. En este punto es interesante tener en cuenta que la formación permanente para los docentes en torno al nuevo programa, en el caso de los maestros rurales, deberá girar en torno a la actualización en las disciplinas y al mismo tiempo, la actualización con respecto a fenómenos de la realidad rural actual que sirvan como anclaje inicial para el tratamiento de aquellos contenidos. Los maestros rurales estarán en inmejorables condiciones para dar cuenta de esa realidad, conformándose instancias de formación permanente a modo de intercambio entre docentes y técnicos, según diversas modalidades: encuentros, talleres; a nivel regional, a nivel departamental, según agrupamientos escolares, a través de la gestión de los maestros coordinadores, Capder, etc.

Desde ese lugar el docente podrá realizar una mirada al Programa en función de las actividades didácticas que tendrá que organizar, incorporando en esa mirada no sólo el texto curricular, sino también todos los elementos ambientales necesarios, las propias convicciones y todos aquellos insumos propios de la didáctica multigrado y de la tradición histórica de la escuela rural referida a saberes y recursos. Ahora las decisiones sobre organización de contenidos en torno a temas de la cotidianeidad estará complejizada y enriquecida por un currículum conceptualmente más denso y disciplinarmente más estructurado.

\section{Lo que es posible hacer}

Así como "seres y fenómenos de la naturaleza" (CNEPyN, 1950, p. 19) constituía un paso hacia lo didáctico, formulado en la estructura curricular; también lo eran las sugerencias planteadas tanto en el Programa para Escuelas rurales como en el Programa para Escuelas urbanas. Desprovista la nueva estructura de todo elemento de esa naturaleza, las acciones desde lo didáctico, deberían tener al menos dos niveles: uno epistemológico y otro de planificación didáctica. Ambos son niveles didácticos, en tanto suponen el manejo y procesamiento de los contenidos a ser enseñados.

Una posibilidad de cumplir con el nivel epistemológico de trabajo es organizar los contenidos en forma de redes. Éstas pueden estructurarse en torno a temas estructuradores, que estén o no en el Programa, que sean susceptibles de integrar contenidos pertenecientes a diferentes áreas y disciplinas y también pertenecientes a diferentes grados, para su comprensión. En el caso de la escuela rural, los contenidos pueden agruparse en torno a la contaminación del arroyo, la forestación, las nuevas técnicas de producción, el invernáculo, las actividades sociales de la comunicación; pero también a temáticas más amplias y lejanas: las que surgen a través de los medios de comunicación, los juegos olímpicos, el calentamiento global, los conflictos políticos internacionales, la crisis financiera, etc.

En todos los casos, se trata de grandes temas para cuyo abordaje es necesaria la concurrencia de muchos contenidos, aunque es decisión del docente limitar la red a la 
concurrencia de unos pocos, para hacer de la red una herramienta viable para la enseñanza de aquellos. Los contenidos allí ubicados deben pertenecer a diferentes disciplinas, en tanto no hay tema de la realidad que pueda ser comprendido apelándose a sólo una disciplina. No ocurre en la ciencia, tampoco debiera ocurrir en la educación. Cada disciplina observa una pequeña porción del fenómeno y es la participación de varias, la que permite una apreciación más global y comprensiva del fenómeno. En el caso de la escuela rural además, los contenidos ubicados en la red pertenecen a diferentes grados, ya que el tema que motiva la red será trabajado por todo el grupo multigrado, aunque cada grado o nivel desde un contenido distinto. De ese modo el tema de una nueva técnica de producción que esquilma los suelos del lugar puede ser abordado desde diferentes contenidos y disciplinas: la geología, el paisaje moldeado por factores internos como el vulcanismo y factores externos como la meteorización y erosión; la economía, tecnificación de la producción con menos mano de obra y mayor uso de máquinas y procesos mecanizados; la biología, una nueva especie vegetal que consume mucha agua o determinado nutriente del suelo; la física y la química, para comprender los fenómenos de meteorización química y mecánica, etc.

Aunque no necesariamente deban consignarse las disciplinas en la red, sí ubicamos allí los contenidos seleccionados en la oportunidad para abordar el tema. La red permitirá contar con distintas puertas de entradas y diferentes caminos internos para abordar la temática, de acuerdo a los grados presentes en el grupo. En ocasiones se aplicará un criterio de complementariedad entre los contenidos trabajados en diferentes grados, en tanto esa es la relación que se mantiene entre ellos. Cada grado se apropia del contenido correspondiente, ayudando a que todos comprendan la temática general. En otras ocasiones, el criterio es de diferente profundización de un mismo contenido, el cual es abordado por todos, pero desde distintos puntos de vista y grados de dificultad.

Una vez definida la organización de contenidos bajo la forma de red y que seleccionemos las entradas y recorridos para grado o nivel del grupo multigrado; comienza el segundo nivel didáctico que supone la planificación de actividades. La planificación periódica - proyectos, centros de interés, núcleos temáticos, tópicos - toman como insumo los temas y contenidos organizados en la red. Allí se traduce esa organización en una temporalidad materializada en actividades que incorpora el resto de los elementos de la práctica educativa que debemos prever: organización de los tiempos y espacios, recursos didácticos a utilizar, secuencia didáctica prevista, organización social de la clase y relaciones interactivas y forma de evaluación.

En la planificación periódica el tema estructurador de la red sigue siendo para todos los grados; pero el plan supone la elaboración de secuencias a ser ejecutadas en un período de tiempo, de carácter complementario, paralelo y de ejecución simultánea. La cantidad de secuencias simultáneas por cada plan periódico dependerá de la cantidad de grados o niveles que estén presentes. Esas secuencias prevén aperturas y puestas en común parciales o finales, donde todo el grupo multigrado asiste conjuntamente a la actividad como grupo total. En los trabajos vinculados a la didáctica multigrado hemos señalado que en esas puestas en común está gran parte del aprovechamiento del potencial del grupo multigrado para los procesos de aprendizaje.

Se trata de poner en común los saberes que cada grado trabaja, de tal forma que puedan circular, trascendiendo las barreras formales de la estructura por grado. Se ubica 
al saber en el lugar central, dejando que circule libremente y cada niño, más allá del grado en que se encuentra, tome de él lo necesario y posible, sin perjuicio de las exigencias mínimas que debe cumplir para el grado en el que se encuentra. Pero el multigrado ofrece la posibilidad de no encasillarse en limitaciones formales, dejando lugar a la ocurrencia de fenómenos sorprendentes en las aulas. La planificación semanal o diaria tendrá como insumos las secuencias establecidas en los planes periódicos y podrá seguir la misma estructura de aquellos, aunque referidas en este caso a las actividades didácticas propiamente dichas.

\section{Reflexiones finales}

El currículum prescripto es el resultado de un proceso donde se tensan poderes e intereses diversos. El producto final representa todas las realidades y a ninguna en particular. En este sentido, una reforma curricular supone un hecho trascendente a tener en cuenta. Mal o bien representa lo que la sociedad elige para transmitir a las nuevas generaciones. En tiempos de escasa materialidad y consistencia del conocimiento, donde su obsolescencia rápidamente deja lugar a otros, en una cadena vertiginosa; adquiere mucha importancia la generación de un texto curricular. Aunque su estabilidad y completitud sean sólo ilusiones, el texto curricular tiene el valor de materializar los saberes elegidos para ser enseñados, construido como una referencia fija a la que volver una y otra vez.

Pero sin ánimo de relativizar más de la cuenta su importancia, está claro que sólo con la estructura curricular no hacemos nada. Un nuevo programa no es garantía de cambios sustanciales en las relaciones educativas. Se necesita formar a los docentes y poner a disposición una serie de insumos que permitan incluir el Programa en las manifestaciones didácticas cotidianas. El nuevo Programa permite y exige mucho trabajo a su alrededor. Su adaptación a la realidad de los grupos multigrado y de los ámbitos rurales, demandará acciones de desarmado y rearmado, desconstrucción y reconstruíción permanentes. Sólo así sus potencialidades serán complementadas y sus carencias serán compensadas por el valor social y didáctico que la pedagogía de la escuela rural tiene para ofrecer.

Ese es el desafío. El desafío pasa por seguir comprometidos con una matriz en la que los maestros se siguen sintiendo representados, pero utilizándola como categoría de análisis para leer la realidad de hoy, bien distinta a la realidad de 1949. Una realidad para la cual aquellas categorías conceptuales siguen siendo útiles. Se ha producido, sin embargo, un proceso de decantación en el que finalmente, por la vía de los hechos, se ha materializado aquella cuestión de la escuela sola no puede, aquella cuestión de la escuela actuando en conjunto con otras instituciones. En las experiencias compartidas en los coloquios, en todos los casos, siempre se dio cuenta de una enorme cantidad de instituciones que, trabajando en conjunto con la escuela, han hecho posible esas experiencias. Ninguna experiencia se desarrolla con la escuela trabajando en solitario, desconectada de otras instituciones que, aunque no estén físicamente, lo están de todos modos. Es claro cómo las redes institucionales, de las cuales tanto se habla pero a veces tan difícil es concretarlas en la práctica, operan detrás de esas experiencias. La escuela, al igual que en aquel concepto de 1949, al igual que lo que se llevó a la práctica en La Mina, era y es el centro de confluencia, el centro que coordina ese esfuerzo interinsti- 
tucional, el centro de referencia. Allí sí la escuela tiene el deber de incidir sobre la comunidad. En los coloquios se notó cómo lo social incidió en todas las experiencias, aun en aquellas excesivamente técnicas o en las estrictamente didácticas.

La ruralidad de hoy es algo que se ha complejizado. Entender qué es lo rural hoy no es tan sencillo porque, sin duda, no hay una ruralidad sino múltiples ruralidades. No hay una escuela rural, hay múltiples escuelas rurales. Ya Julio Castro en La Escuela rural en el Uruguay (1944) señalaba las diferencias que existían entre el medio rural ganadero del norte y el medio rural agrícola del sur, y cómo la idiosincrasia y la lógica de las escuelas rurales enclavadas en uno y otro medio eran bien diferentes. Ya en ese momento no se hablaba de una sola ruralidad. Hoy que se ha complejizado el medio rural, se hace más difícil caracterizarlo, pero también se hace más difícil identificar al medio rural como separado del medio urbano. Las fronteras, los límites, se desdibujan. Hoy en día se producen procesos de ruralización de los medios urbanos y de urbanización de los medios rurales. Hay fenómenos, producto de los procesos demográficos, productivos y culturales, que tienen como efecto, trabajadores rurales que no viven en el medio rural y se desplazan hacia allí desde el pueblo. También hay lógicas de funcionamiento en las que los pequeños pueblos tienen una impronta rural, aunque puedan ser catalogados demográficamente como medios urbanos. Hay una penetración por los medios de comunicación y transporte de pautas culturales y sociales de lo urbano en el medio rural. Las fronteras se han desdibujado y eso tiene que ver con una realidad de la cual hay que dar cuenta. Y allí está la escuela rural, ubicada en esa tensión.

Visualizar el rol de la escuela rural en este marco de la complejidad del mundo actual implica sostener algunas cuestiones de las cuales hay cierta convicción, mínimas pero potentes, que son producto de la herencia pedagógica y que está a flor de piel en los maestros de todo el país. Aun cuando el magisterio rural ha pasado por una etapa crítica de la cual se quiere restablecer y a partir de la cual reconstruir el terreno perdido. En esa etapa los maestros han tenido una formación específica muy desdibujada. La formación inicial de los maestros se ha diluido en una generalidad, no reconociéndose por parte de las políticas educativas, la especificidad rural que los docentes nunca dejaron de defender. Pero aun con esas condicionantes, en el magisterio rural fluye por los poros esa herencia que está presente y no puede dejar de estarlo. Sobre la base de esa herencia, sobre los conceptos que implica, es que se puede construir la lectura de estos tiempos e imaginar escenarios futuros posibles.

\section{Referencias}

CNEPyN. Fundamentos, concepto y fines de la escuela rural. Anales de Instrucción Primaria, 1949.

CNEPyN. Programa para escuelas rurales. Montevideo: Imprenta Nacional, 1950.

CASTRO, Julio. La escuela rural en el Uruguay. Montevideo: Imprenta Nacional, 1944.

SANTOS, Limber. El nuevo programa: miradas desde la escuela rural. Quehacer Educativo, n. 93, 2009, p. 215-222.

SANTOS, Limber. El campo pedagógico en Suma Sarnaqaña. Montevideo: Colectivo Suma Sarnaqaña, 2011.

SOLER, Miguel. Réplica de um maestro agredido. Montevideo: Trilce, 2005. 
LIMBER ELBIO SANTOS es maestro y licenciado en Ciencias de la Educación. Director nacional del Departamento de Educación para el Medio Rural, Consejo de Educación Inicial y Primaria, Administración Nacional de Educación Pública, Uruguay. Docente del Departamento de Historia y Filosofía de la Educación y del Departamento de Enseñanza y Aprendizaje, Universidad de la República, Uruguay. Contacto: Peluffo e Pargas y 25 de agosto - Covipa III, 344 - Pando 91000 Canelones - Uruguay.

E-mail: limbersantos@gmail.com.

Recebido em 23 de maio de 2013.

Aceito em 8 de outubro de 2013. 